\title{
Estimating Differential Quantities using Polynomial fitting of Osculating Jets *i
}

\author{
F. Cazals $\stackrel{\ddagger}{\ddagger}$ M. Pouget ${ }^{\S}$
}

September 20, 2004

\begin{abstract}
This paper addresses the point-wise estimation of differential properties of a smooth manifold $S$-a curve in the plane or a surface in $3 D$ - assuming a point cloud sampled over $S$ is provided. The method consists of fitting the local representation of the manifold using a jet, and either interpolation or approximation. A jet is a truncated Taylor expansion, and the incentive for using jets is that they encode all local geometric quantities — such as normal, curvatures, extrema of curvature.

On the way to using jets, the question of estimating differential properties is recasted into the more general framework of multivariate interpolation / approximation, a well-studied problem in numerical analysis. On a theoretical perspective, we prove several convergence results when the samples get denser. For curves and surfaces, these results involve asymptotic estimates with convergence rates depending upon the degree of the jet used. For the particular case of curves, an error bound is also derived. To the best of our knowledge, these results are among the first ones providing accurate estimates for differential quantities of order three and more. On the algorithmic side, we solve the interpolation/approximation problem using Vandermonde systems. Experimental results for surfaces of $\mathbb{R}^{3}$ are reported. These experiments illustrate the asymptotic convergence results, but also the robustness of the methods on general Computer Graphics models.
\end{abstract}

Keywords. Meshes, Point Clouds, Differential Geometry, Interpolation, Approximation.

\section{Introduction}

\subsection{Estimating differential quantities}

Several applications from Computer Vision, Computer Graphics, Computer Aided Design or Computational Geometry require estimating local differential quantities. Examples of such applications are surface segmentation, surface smoothing / denoising, surface reconstruction, shape design. In any case, the input consists of a point cloud or a mesh. Most of the time, estimating first and second order differential quantities, that is the tangent plane and curvature-related quantities, is sufficient. However, some applications involving shape analysis require estimating third order differential quantities $\left[\mathrm{HGY}^{+} 99\right.$, Por01].

Given these ubiquitous needs, a wealth of different estimators have been proposed in the vast literature of applied geometry [Pet01, GI04]. Most of these are adaptations to the discrete setting of smooth differential geometry results. For example, several definitions of normals, principal directions and curvatures over a mesh can be found in [Tau95, CW00]. Ridges of polyhedral surfaces as well as cuspidal edges of the focal sets are computed in [WB01]. Geodesics and discrete versions of the Gauss-Bonnet theorem are considered in [PS98].

Out of all these contributions, few of them address the question of the accuracy of the estimates proposed or that of their convergence when the mesh or the sample points get denser. This lack of sound theoretical analysis is however a major issue since discrete versions of smooth operators may not converge, or may converge to unexpected values. Examples of such phenomena are the surface area of a mesh which may not converge to that of the

\footnotetext{
${ }^{*}$ An extended abstract of this paper is part of the Symposium on Geometry Processing (SGP), 2003. This paper provides the proofs of the SGP paper and also features an enhanced experimental section.

†ork partially supported by the European Project Effective Computational Geometry for Curves and Surfaces, Shared-cost RTD (FET Open) Project No IST-2000-26473

†INRIA Sophia-Antipolis, 2004 route des Lucioles, F-06902 Sophia-Antipolis; Frederic.Cazals@inria.fr

§INRIA Sophia-Antipolis, 2004 route des Lucioles, F-06902 Sophia-Antipolis; Marc.Pouget@inria.fr
} 
discretized surface [MT02], or the angular defect at a vertex of a triangulation which usually does not provide any information on the Gauss curvature of the underlying smooth surface [BCM02].

The estimation methods providing convergence guarantees are all concerned with first and second order differential quantities. In [AB99], an error bound is proved on the normal estimate to a smooth surface sampled according to a criterion involving the skeleton. The surface area of a mesh and its normal vector field versus those of a smooth surface are considered in [MT02]. Asymptotic estimates for the normal and the Gauss curvature of a sampled surface for several methods are given in [MW00]. Based upon the normal cycle and restricted Delaunay triangulations, an estimate for the second fundamental form of a surface is developed in [CSM03].

Another striking fact about the estimation of first and second order differential quantities is that for plane curves, these quantities are often estimated using the osculating circle, while for surfaces, using osculating paraboloids are ubiquitous. Why not osculating parabolas for curves and osculating ellipsoids or hyperboloids for surfaces? Answering this question and developing estimation methods providing guarantees for third and higher order estimates motivates the following contributions.

\subsection{Contributions}

The main contribution of this paper is to recast the problem of estimating differential properties to that of fitting the local representation of the manifold by a jet. A jet is a truncated Taylor expansion, and the incentive for using jets is that they encode all local geometric quantities - such as normal, curvatures, extrema of curvature. Accurate estimates of the coefficients of the jet therefore translate immediately to accurate estimates of differential quantities. Since the method proposed consists of performing a polynomial fitting, connections with the classical questions of interpolation and approximation deserve a careful discussion.

Interpolation is a well studied topics in numerical analysis. Most of the time however, the parameterization domain of interest is a subset of $\mathbb{R}^{n}$. In particular, convergence results on the coefficients of the Lagrange interpolation polynomial versus the Taylor expansion of a function are proved in [Coa66, CR72, QV94]. Our results differ from these in several concerns. On one hand, we are interested in interpolation and approximation over a manifold rather than a Euclidean domain. On the other hand, the afore-mentioned papers prove error bounds while we establish asymptotic error estimates. It should however be noticed that the dominant terms of these - as a function of the sampling density - are the same.

Regarding polynomial fitting of differential properties for a surface, our results are closely related to [MW00, Lemma 4.1]. In that paper, a degree two interpolation is used and analyzed. We generalize this result for jets or arbitrary degree, under interpolation and approximation.

To complete the description, two comments are in order. First, it should emphasized that our focus is a local interpolation / approximation problem, that is we are not concerned with the convergence of the Lagrange interpolation polynomial to the height function on a whole given set. This problem requires specific conditions on the function and the position of the points, as illustrated by the Runge divergence phenomena [LS86, Chapter 2]. Therefore, our study is not to be confused with global fitting such as piecewise polynomial fitting encountered in CAD. Second, our focus is on the estimation of point-wise differential quantities and not the identification of loci of points exhibiting prescribed differential properties - examples of such loci are ridges or parabolic lines. While the former problem is local, the latter is global and therefore faces the issue of reporting loci with a global topological coherence [Por01, CP04].

\subsection{Paper overview}

Fundamentals about jets and numerical issues are recalled in sections 2 and 3 . The case of surfaces and curves are examined in sections 4 and 5. Finally, the overall algorithm together with experimental results are presented in sections 6 and 7. 


\section{Geometric pre-requisites}

\subsection{Curves and surfaces, height functions and jets}

It is well known [dC76, Spi99] that any regular embedded smooth ${ }^{1}$ curve or surface can be locally written as the graph of a univariate or bivariate function with respect to any $z$ direction that does not belong to the tangent space. We shall call such a function a height function. Taking an order $n$ Taylor expansion of the height function over a curve yields:

$$
f(x)=J_{B, n}(x)+O\left(x^{n+1}\right)
$$

with

$$
J_{B, n}(x)=B_{0}+B_{1} x+B_{2} x^{2}+B_{3} x^{3}+\ldots+B_{n} x^{n}
$$

Similarly for a surface:

$$
f(x, y)=J_{B, n}(x, y)+O\left(\|(x, y)\|^{n+1}\right),
$$

with

$$
J_{B, n}(x, y)=\sum_{k=0}^{n} H_{B, k}(x, y), H_{B, k}(x, y)=\sum_{j=0}^{k} B_{k-j, j} x^{k-j} y^{j} .
$$

Notice that in Eq. (3), the term $O\left(\|(x, y)\|^{n+1}\right)$ stands for the remainder in Taylor's multivariate formula.

Borrowing to the jargon of singularity theory [BG92], the truncated Taylor expansion $J_{B, n}(x)$ or $J_{B, n}(x, y)$ is called a degree $n$ jet, or $n$-jet. Since the differential properties of a $n$-jet match those of its defining curve/surface up to order $n$, the jet is said to have a $n$ order contact with its defining curve or surface. This also accounts for the term osculating jet -although osculating ${ }^{2}$ was initially meant for 2 -jets. The degree $n$-jet of a curve involves $n+1$ terms. For a surface, since there are $i+1$ monomials of degree $i$, the $n$-jet involves $N_{n}=1+2+\cdots+(n+1)=$ $(n+1)(n+2) / 2$ terms. Notice that when the $z$ direction used is aligned with the normal vector to the curve/surface, one has $B_{1}=0$ or $B_{1,0}=B_{0,1}=0$. The osculating $n$-jet encloses differential properties of the curve/surface up to order $n$, that is any differential quantity of order $n$ can be computed from the $n$-jet. In particular, the tangent space can be computed from the 1 -jet, the curvature related information can be obtained from the 2 -jet, .... To clarify the presentation, we summarize as follows:

Definition. 1 For a given point on a curve or surface and $n \geq 1$ :

- given a coordinate system, the osculating $n$-jet is the Taylor expansion of the height function truncated at order $n$.

- the osculating $n$-jet is principal in a given coordinate system if the linear terms vanish (i.e. the z-axis is the normal direction of the manifold). Note that this is rather a property of the coordinate system which reads on the jet.

- an osculating conic/quadric is a conic/quadric whose 2-jet matches that of the curve/surface (independently of a given coordinate system).

- an osculating conic/quadric is degenerate if the quadratic form this conic/quadric is defined with is degenerate (that is the form does not have full rank).

- an osculating conic/quadric is principal if, in the given coordinate system, its n-jet are principal.

Degenerate osculating conics/quadrics are specific curves and surfaces since:

Theorem. 1 [Ber87, Chapter 15] There are 9 Euclidean conics and 17 Euclidean quadrics.

Observation. 1 The degenerate osculating conics to a smooth curve are parabola or lines. The degenerate osculating quadrics to a smooth surface are either paraboloids (elliptic, hyperbolic), parabolic cylinders, or planes.

\footnotetext{
${ }^{1}$ Regular means that the tangent space has dimension one/two for a curve/surface everywhere. Embedded forbids self-intersections. Smooth means as many times differentiable as we need, typically $C^{3}$ or $C^{4}$.

${ }^{2}$ From the Latin osculare, to kiss.
} 
Degenerate osculating conics and quadrics are therefore respectively 2 out of 9 conics and 4 out of 17 quadrics.

The Monge coordinate system of a curve is defined by its tangent and normal vectors. For a surface, the Monge coordinate system is such that the $z$ axis is aligned with the normal and the $x, y$ axis are aligned with the principal directions. Note that the $n$-jet is principal in the Monge coordinate system. The Monge form of the curve or surface at one of its point is the local Taylor expansion of the curve/surface in the Monge coordinate system. In this particular system, the height function is called the Monge form, and letting $k_{1}, k_{2}$ stand for the principal curvatures, one has

$$
f(x, y)=\frac{1}{2}\left(k_{1} x^{2}+k_{2} y^{2}\right)+O\left(\|(x, y)\|^{3}\right) .
$$

From these observations, the question we ended paragraph 1.1 with can now be answered. By theorem 1 and observation 1, using a general conic/quadric or a degenerate one to approximate a curve or a surface does not make any difference. In both case and up to order two, the local differential properties of the curve/surface, degenerate conic/quadric, or full rank conic/quadric are identical. All these local differential properties are enclosed is the 2-jet of the manifold. Yet, from a practical point of view, finding a conic/quadric requires more constrains than finding a degenarate one since the latter reduces to a degree two polynomial fitting. Notice also that the normal direction to the manifold being a priori unknown, this polynomial fitting has to be performed in a coordinate system where the osculating jet is (in general) not principal.

As an example, consider Figure 1(a,b,c,d). Figure 1(a) features a curve $C$ and an osculating conic ${ }^{3}$. In (b), the osculating circle is drawn, in (c), the osculating circle is replaced by the principal osculating parabola - whose symmetry axis is the normal to $C$ and whose curvature matches that of $C$. At last, in (d) a general parabola locally approximates $C$ in a coordinate system where the jet is not principal (its symmetry axis is not aligned with the normal to $C$ ). This last case illustrates the setting we are working with in the sequel.
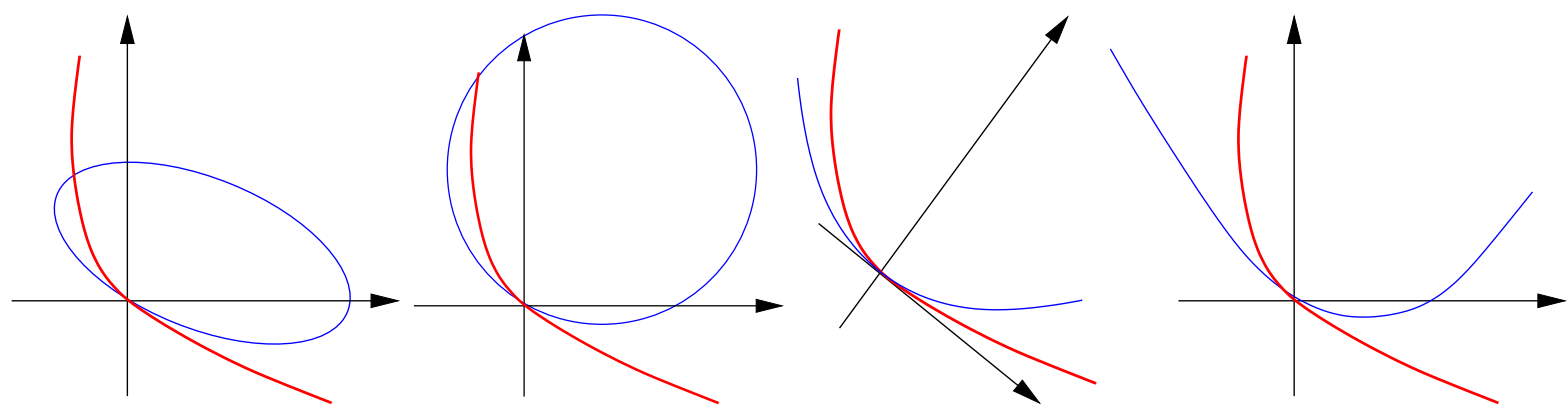

Figure 1: A curve and (a) an osculating ellipse, (b) its osculating circle (special case of osculating conic), (c) its principal degenerate conic, (d) an degenerate osculating conic: a parabola.

\subsection{Interpolation, approximation and related variations}

Our methodology to retrieve differential quantities consists of fitting the osculating jet. The following variations need to be discussed in order to state our contributions precisely. The case of curves and surfaces being tantamount, our description focuses on surfaces. Assume we are given a set of $N$ points $p_{i}\left(x_{i}, y_{i}, z_{i}\right), i=1, \ldots, N$ in the neighborhood of a given point $p$ on the surface processed. Point $p$ itself may or may not be one of the $N$ samples, and one can assume without loss of generality that $p$ is located at the origin of the coordinate system used.

Interpolation versus approximation. Interpolating consists of finding a polynomial that fits exactly a set of data points. In our case and following Equation (3), let $B$ index a coefficient of the jet of the surface, and $A$ index a coefficient of the jet sought ${ }^{4}$. We aim at finding a $n$-jet $J_{A, n}$ such that

$$
f\left(x_{i}, y_{i}\right)=J_{B, n}\left(x_{i}, y_{i}\right)+O\left(\left\|\left(x_{i}, y_{i}\right)\right\|^{n+1}\right)=J_{A, n}\left(x_{i}, y_{i}\right), \quad \forall i=1, \ldots, N .
$$

\footnotetext{
${ }^{3} \mathrm{~A}$ point worth noticing is the relative position of $C$ and an osculating curve: the former usually crosses the latter at the intersection point. To see why, compare the order three Taylor expansions of the of two curves.

${ }^{4}$ As a mnemonic, the reader may want to remind that index $A$ stands for the Answer to the fitting problem.
} 
Approximation, on the other hand, gives up on exactness, that is the graph of the jet sought may not contain the sample points. We shall focus on least-square approximation, which consists of minimizing the sum of the square errors between the value of the jet and that of the function. The quantity to be minimized is therefore

$$
\sum_{i=1}^{N}\left(J_{A, n}\left(x_{i}, y_{i}\right)-f\left(x_{i}, y_{i}\right)\right)^{2} \text {. }
$$

The two problems can actually be written in the same matrix form. To see why, let us write the jets in the polynomial basis consisting of monomials $x^{i} y^{j}$. Examples of other basis that could be used are the Bezier-Bernstein basis or the Newton basis. We use the monomials since this basis is convenient for the asymptotic analysis but also the design of effective algorithms. Denote $A$ the $N_{n}$-vector of the coefficients of the jet sought, that is

$$
A=\left(A_{0,0}, A_{1,0}, A_{0,1}, \ldots, A_{0, n}\right)^{t} .
$$

Denote $Z$ the $N$-vector of the ordinates, i.e. with $z_{i}=f\left(x_{i}, y_{i}\right)$,

$$
Z=\left(z_{1}, z_{2}, \ldots, z_{N}\right)^{t}=\left(J_{B, n}\left(x_{i}, y_{i}\right)+O\left(\left\|\left(x_{i}, y_{i}\right)\right\|^{n+1}\right)\right)_{i=1, \ldots, N} .
$$

Equations (6) and (7) yield the following $N \times N_{n}$ Vandermonde matrix

$$
M=\left(1, x_{i}, y_{i}, x_{i}^{2}, \ldots, x_{i} y_{i}^{n-1}, y_{i}^{n}\right)_{i=1, \ldots, N} .
$$

For the interpolation case, the number of points matches the number of parameters, so that matrix $M$ is square and Eq. (6) can be written as $M A=Z$. For the approximation case, $M$ is a rectangular $N \times N_{n}$ matrix, and Eq. (7) is summarized as $\min \|M A-Z\|_{2}$.

Choosing between interpolation and approximation depends upon the problem tackled. For noisy datasets, approximation is the method of choice. Otherwise, the alternative depends of the relative values of the number of model parameters versus the number of available points. If the two match one-another, a natural choice is interpolation. In any case, fitting yields a linear system, so that numerical issues arise. Facing these difficulties is the topic of section 3 .

Mesh or mesh-less methods. An important difference between local geometry estimation algorithms is whether or not they require some topological information - typically the connectivity of a mesh. Mesh-based methods are usually faster. Mesh-less techniques are more general and better suited for noisy datasets. A difficulty of the latter methods, however, is to select the relevant points used to perform the estimates. While one can always resort to heuristics of the $k$-nearest-neighbors type, user defined parameters should be avoided. This issue is addressed in section 6.

One or two stages methods. Fitting a 2-jet provides estimates of the tangent plane and the curvature related information. These steps can be carried out sequentially or simultaneously. Following the guideline of [SZ90], most of the methods already mentioned proceed sequentially. The provably good algorithm we propose proceeds simultaneously. Along its analysis, we also provide theoretical results on the accuracy of sequential methods.

\subsection{Contributions revisited}

Equipped with the language of jets, we can now state our contributions precisely. Consider Eqs. (6) and (7). We expect $J_{A, n}$ and $J_{B, n}$ to be equivalent in some sense. To specify this, we shall study the convergence properties of the coefficients of $J_{A, n}$ when the points $p_{i}$ converge to $p$. More precisely, assume that the coordinates of the $p_{i}$ are given by $p_{i}\left(x_{i}=a_{i} h, y_{i}=b_{i} h, z_{i}=f\left(x_{i}, y_{i}\right)\right)$. Parameters $a_{i}$ and $b_{i}$ are arbitrary real numbers, while $h$ specifies that the $p_{i}$ uniformly tend to the origin. We actually expect

$$
A_{i j}=B_{i j}+O(r(h))
$$

Function $r(h)$ describes the convergence rate or the precision of the fitting, and the main contribution of this paper is to quantify $r(h)$ for interpolation and approximation methods. This is done applying classical results of numerical analysis. These results are then translated for geometric quantities such as normal and curvatures. As we shall see, interpolation or approximation of the same degree yield the same convergence rate. The difficulties posed are also similar and are essentially to deal with singular matrices. This enable the design of an algorithm for the estimation of geometric quantities together with some knowledge about the quality of these estimates. 


\section{Numerical pre-requisites}

In this section, we recall the fundamentals of the fitting methods used, namely interpolation and approximation, together with the numerical issues arising from the resolutions.

\subsection{Interpolation}

The interpolation fitting is based upon the Lagrange interpolation, that is the construction of a polynomial constrained to fit a set of data points. Although this problem is classical for the univariate case, the multivariate case is still an active research field from both the theoretical and computational points of view. We briefly review the univariate and multivariate basics of Lagrange interpolation.

Univariate Lagrange interpolation. Let $X=\left\{x_{0}, \ldots, x_{n}\right\}$ be $n+1$ distinct real values, the so-called nodes. Then, for any real function $f$, there is a unique polynomial $P$ of degree $n$ so that $P\left(x_{i}\right)=f\left(x_{i}\right), \forall i=0, \ldots, n$. Polynomial $P$ is called the Lagrange interpolation polynomial of $f$ at the nodes $X$. For any choice of distinct nodes, this polynomial exists and is unique, and in that case the Lagrange interpolation is said to be poised.

Multivariate Lagrange interpolation. Consider now the following bivariate problem. Let $\Pi_{n}$ be the subspace of bivariate polynomials of total degree equal or less than $n$, whose dimension is $N_{n}=\left(\begin{array}{c}n+2 \\ n\end{array}\right)$, and let $X=\left\{x_{1}, \ldots, x_{N}\right\}$ consist of $N=N_{n}$ values in $\mathbb{R}^{2}$ called nodes. (Notice that $N$ is exactly the number of monomials found in the jet of Equation 3.) The Lagrange interpolation problem is said to be poised for $X$ if for any function $f: \mathbb{R}^{2} \rightarrow \mathbb{R}$, there exists a unique polynomial $P$ in $\Pi_{n}$ so that $P\left(x_{i}\right)=f\left(x_{i}\right), \forall i=1, \ldots, N$. It is intuitive and well known that this problem is poised iff the set of nodes $X$ is not a subset of any algebraic curve of degree at most $n$, or equivalently the Vandermonde determinant formed by the interpolation equations does not vanish. As noticed in [SX95], the set of nodes for which the problem is not poised has measure zero, hence it is almost always poised.

However let us illustrate non-poised cases or degenerate configurations of nodes, together with almost degenerate configurations - a more precise definition will be given with the conditioning in section 3.3. Consider the two quadrics $q_{1}(x, y, z)=2 x+x^{2}-y^{2}-z$ and $q_{2}(x, y, z)=x^{2}+y^{2}-z$, whose intersection curve $I$ projects in the $(x, y)$ plane to the conic $C(x, y)=0$ with $C(x, y)=x-y^{2}$ (cf. Fig. 2). If one tries to interpolate a height function using points on $I$, uniqueness of the interpolant is not achieved since any quadric in the pencil of $q_{1}$ and $q_{2}$ goes through $I$. A similar example featuring the four one-ring and one two-ring neighbors of a point $p$ is depicted on figure 3. Notice that being able to figure out such configurations is rather a strength than a weakness of the method since a surface is sought and, the amount of information available does not determine uniquely such a surface. A first fundamental difference between the univariate and multivariate cases is therefore the critical issue of choosing nodes so that the interpolation is poised.

In the particular case where the points lies on a regular square grid of the plane, the geometry of the configuration leads to the following remarks. On one hand, a non-poised degree $n$ interpolation occurs if the points lies on $n$ lines, since they define an algebraic curve of degree $n$. One the other hand, triangular lattices yield poised problems for every degree. These results and further extensions can be found in [GS00] and references therein.

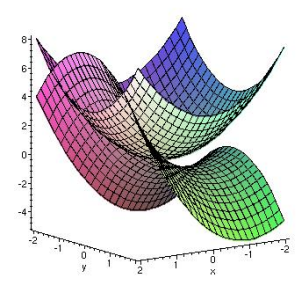

Figure 2: Two quadrics whose intersection curve $I$ projects onto the parabola $C: x=y^{2}$. Interpolation points located on $I$ do not uniquely define an interpolating height function.

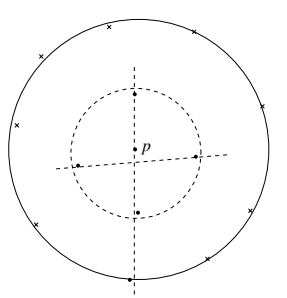

Figure 3: The Kite (almost) degenerate configuration tangent plane seen from above: the 6 points used for a degree two interpolation are (almost) located on a conic, that is two intersecting lines. 


\subsection{Least square approximation}

It is well known that the minimization problem of Eq. (7) has a unique solution iff the matrix $M$ is of maximum rank $N_{n}$. In that case, the minimum value $\rho$ is called the residual of the system, that is $\rho=\min \|M A-Z\|_{2}$. The important issue is again the rank of the matrix $M$. In terms of the relative values of $N$ versus $N_{n}$, using too many points certainly smoothes out geometric features, but also makes rank deficient matrices less likely.

\subsection{Numerical Issues}

The difficulties of solving linear and least-squares systems consist of dealing with rank-deficient matrices. We now discuss these issues in more detail. Distances between matrices and matrix norms refer to the Euclidean norm.

Singular systems and condition numbers. To quantify degeneracies, we resort to a Singular Value Decomposition (SVD) [GvL83]. Denote $\sigma_{n}, \ldots, \sigma_{1}$ the singular values of $M$ sorted in decreasing order. It is well known that the least singular value of $M$ is the distance from $M$ to rank deficient matrices. The singular values also characterizes the sensitivity of the problem, that is the way errors on the input data induce errors on the computed solution. Notice that errors refer to the uncertainty attached to the input data and not to the rounding errors inherent to the floating point calculations. In our case, input data are the sample points, so that errors are typically related to the acquisition system - e.g. a laser range scanner.

To quantify the previous arguments, we resort to the conditioning or condition number of the system [GvL83, Hig96]. The conditioning is defined as a magnification factor which relates the afore-mentioned errors by the following rule Error on solution $=$ Error on input $\times$ conditioning. Denote

$$
\kappa_{2}(M)=\|M\|\left\|_{2}|| M^{-1}\right\|_{2}=\sigma_{n} / \sigma_{1}
$$

the condition number of the matrix $M$. The conditioning of the linear problem $M X=Z$ and the least square problem $\min \|M X-Z\|_{2}$ are respectively given by

$$
\begin{cases}\text { linear square system: } & 2 \kappa_{2}(M), \\ \text { least square system: } & 2 \kappa_{2}(M)+\frac{\kappa_{2}(M)\left(\kappa_{2}(M)+1\right) \rho}{\|M\|_{2}\|X\|_{2}} \text { with } \rho=\|M X-Z\|_{2} \text { the residual. }\end{cases}
$$

The following theorem provides precise error bounds:

Theorem. 2 ([Hig96] p. 133 and 392) Suppose $X$ and $\widetilde{X}$ are the solutions of the problems

$$
\begin{cases}\text { linear square system: } & M X=Z \text { and }(M+\Delta M) \widetilde{X}=Z+\Delta Z, \\ \text { least square system: } & \min \|M X-Z\|_{2} \text { and } \min \|(M+\Delta M) \widetilde{X}-(Z+\Delta Z)\|_{2},\end{cases}
$$

with $\varepsilon$ a positive real value such that $\|\Delta M\|_{2} \leq \varepsilon|| M\left\|_{2},\right\| \Delta Z\left\|_{2} \leq \varepsilon\right\| Z \|_{2}$, and $\varepsilon \kappa_{2}(M)<1$. Then one has:

$$
\frac{\|X-\tilde{X}\|_{2}}{\|X\|_{2}} \leq \frac{\varepsilon}{1-\kappa_{2}(M) \varepsilon} \text { conditioning }
$$

In practice, if the conditioning is of order $10^{a}$ and the relative error on the input is $\varepsilon \approx 10^{-b}-$ with $\varepsilon \kappa_{2}(M)<1$, then the relative error on the solution is of order $10^{a-b}$.

Pre-conditioning the Vandermonde system. As already discussed, a convenient way to solve Eqs. (6) and (7) consists of using the basis of monomials. One ends up with the Vandermonde matrix of Eq. (8), that can be solved with usual methods of linear algebra. Unfortunately, Vandermonde systems are known to be ill-conditioned due to the change of magnitude of the terms. We therefore pre-condition so as to improve the condition number. Assuming the $\left\{x_{i}\right\},\left\{y_{i}\right\}$ are of order $h$, the pre-conditioning consists of performing a column scaling by dividing each monomial $x_{i}^{k} y_{i}^{l}$ by $h^{k+l}$.

The new system is $M^{\prime} Y=M D Y=Z$ with $D$ the diagonal matrix $D=\left(1, h, h, h^{2}, \ldots, h^{n}, h^{n}\right)$, so that the solution $A$ of the original system is $A=D Y$. The condition number used in the sequel is precisely $\kappa\left(M^{\prime}\right)$. (Notice it has the geometric advantage to be invariant under homothetic transformations of the input points.) Then the accuracy of the result can be estimated a posteriori, and almost degenerate cases hight-lighted by large conditioning. 
Alternatives for the interpolation case. An alternative to the Vandermonde system consists of using the basis of Newton polynomials. Resolution of the system can be done using divided differences [Sau95], a numerically accurate yet instable method [Hig96].

\section{Surfaces}

\subsection{Problem addressed}

Let $S$ be a surface and $p$ be a point of $S$. Without loss of generality, we assume $p$ is located at the origin and we aim at investigating differential quantities at $p$. Consider the height function $f$ given by Equation (3) in any coordinate system whose $z$ axis is not in the tangent plane. We shall interpolate $S$ by a bivariate $n$-jet $J_{A, n}(x, y)$ whose graph is denoted $Q$.

The normal to a surface given by Equation (3) is

$$
n_{S}=\left(-B_{10},-B_{01}, 1\right)^{t} / \sqrt{1+B_{10}^{2}+B_{01}^{2}} .
$$

In order to characterize curvature properties, we resort to the Weingarten map $A$ of the surface also called the shape operator, that is the tangent map of the Gauss map. (Recall that the first and second fundamental forms $I, I I$ and $A$ satisfy $I I(v, v)=I(A(v), v)$ for any vector $v$ of the tangent space.) The principal curvatures and principal directions are the eigenvalues (eigenvectors) of $A$, and the reader is referred to [dC76, Section 3.3]. If the $z$ axis is aligned with the normal, the linear terms of Equation (3) vanish, and the second fundamental form reduces to the Hessian of the height function. Further simplifications are obtained in the Monge coordinate system, where $I=I d_{2}$, the Hessian is a diagonal matrix, and the principal curvatures are given by $2 B_{20}$ and $2 B_{02}$.

\subsection{Polynomial fitting of the height function}

We begin by an approximation result on the coefficients of the height function. We focus on the convergence rate given by the value of the exponent of parameter $h$.

Proposition. 1 Let $\left\{\left(x_{i}, y_{i}\right)\right\}_{i=1, \ldots, N}$ be a set of points of $\mathbb{R}^{2}$ defining a poised polynomial interpolation or approximation problem of degree n, with $x_{i}=O(h), y_{i}=O(h)\left(N=N_{n}\right.$ for interpolation and $N>N_{n}$ for approximation). Let $J_{A, n}$ be the polynomial solution of the problem associated to the function $f$ that is, for interpolation

$$
J_{A, n}\left(x_{i}, y_{i}\right)=f\left(x_{i}, y_{i}\right), \forall i=1, \ldots, N
$$

and for approximation

$$
J_{A, n}=\arg \min \left\{\sum_{i=1}^{N}\left(J_{A, n}\left(x_{i}, y_{i}\right)-f\left(x_{i}, y_{i}\right)\right)^{2}\right\} .
$$

Then the coefficients $B_{k-j, j}$ of degree $k$ of the Taylor expansion of $f$ are estimated by those of $J_{A, n}$ up to accuracy $O\left(h^{n-k+1}\right)$ :

$$
A_{k-j, j}=B_{k-j, j}+O\left(h^{n-k+1}\right) \quad \forall k=0, \ldots, n \forall j=0, \ldots, k .
$$

Moreover, if the origin is one of the chosen points and interpolation is used, then $A_{0,0}=B_{0,0}=0$.

Proof. [Proof of Prop. 1, interpolation case.] Let $K$ be the convex hull of the set $\left\{\left(x_{i}, y_{i}\right)\right\}_{i=1, \ldots, N}, d_{\max }$ be the diameter of $K$ and $d_{\min }$ be the supremum of the diameter of disks inscribed in $K$. Also denote $D^{k}$ is the differential of order $k$.

The result is a direct consequence of Theorem 2 of [CR72] or remark 3.4.2 of [QV94], which states that

$$
\sup \left\{\left\|D^{k} f(x, y)-D^{k} J_{A, n}(x, y)\right\| ;(x, y) \in K\right\}=O\left(h^{n-k+1}\right) .
$$


Rephrasing it with our notations yields:

$$
\begin{aligned}
\left|B_{k-j, j}-A_{k-j, j}\right| & =\frac{1}{j !(k-J) !}\left|D^{k}\left(f-J_{A, n}\right)_{(0,0)}(1,0)^{k-j}(0,1)^{j}\right| \\
& \leq \sup \left\{\left|D^{k}\left(f-J_{A, n}\right)_{(0,0)}\left(\zeta_{1}, \ldots, \zeta_{k}\right)\right| ; \zeta_{i} \in \mathbb{R}^{2},\left\|\zeta_{i}\right\| \leq 1\right\} \\
& \leq\left\|D^{k}\left(f-J_{A, n}\right)_{(0,0)}\right\| \\
& \leq \sup \left\{\left\|D^{k} f(x, y)-D^{k} J_{A, n}(x, y)\right\| ;(x, y) \in K\right\} .
\end{aligned}
$$

For the particular case where the origin is one of the samples, notice that the equation involving the point $(0,0)$ is $A_{0,0}=f(0,0)=0$.

As outlined by the proof, the constant hidden in the term $O\left(h^{n-k+1}\right)$ depends upon $\sup \left\{\left\|D^{n+1} f(x, y)\right\| ;(x, y) \in\right.$ $K\}$, and the geometry of $K$. In particular, the estimates are better when the ratio $d_{\max } / d_{\min }$ is small, which intuitively means that the set $K$ is not too "flat".

For the approximation case, the result might be a consequence of the theorem 5 of [CR72] formulated in the Sobolev setting. But in order to meet its hypothesis, one must prove that the operator of discrete least square approximation is continuous, which is not straightforward. Alternatively, we give the following pedestrian proof.

Proof. [Proof of Prop. 1, approximation case.]

Using the notations introduced in section 2.2, consider the least-square system $\min \|M A-Z\|_{2}$. With the assumption that $M$ is of rank $N_{n}$, the approximation is equivalent to the invertible linear system $M^{T} M A=M^{T} Z$. With the notation $\Sigma_{l}^{k}=\sum_{i=1}^{N} x_{i}^{k} y_{i}^{l}$ and the assumption $\left\|\left(x_{i}, y_{i}\right)\right\|=O(h)$ :

$$
\begin{aligned}
& M^{T} Z=\left(\begin{array}{lr}
B_{0,0} \Sigma_{0}^{0}+B_{1,0} \Sigma_{0}^{1}+B_{0,1} \Sigma_{1}^{0}+\ldots & +B_{0, n} \Sigma_{n}^{0}+O\left(h^{n+1}\right) \\
B_{0,0} \Sigma_{0}^{1}+\ldots & +B_{0, n} \Sigma_{n}^{1}+O\left(h^{n+2}\right) \\
B_{0,0} \Sigma_{1}^{0}+\ldots & +O\left(h^{n+2}\right) \\
\vdots & \vdots \\
B_{0,0} \Sigma_{n}^{0}+\ldots & +O\left(h^{n+n+1}\right)
\end{array}\right) \\
& M^{T} M=\left(\begin{array}{ccccc}
\Sigma_{0}^{0} & \Sigma_{0}^{1} & \Sigma_{1}^{0} & \ldots & \Sigma_{n}^{0} \\
\Sigma_{0}^{1} & \Sigma_{0}^{2} & \Sigma_{1}^{1} & \ldots & \Sigma_{n}^{1} \\
\Sigma_{1}^{0} & \Sigma_{1}^{1} & \Sigma_{2}^{0} & \ldots & \Sigma_{n+1}^{0} \\
\vdots & \vdots & \vdots & \vdots & \vdots \\
\Sigma_{n}^{0} & \Sigma_{n}^{1} & \Sigma_{n+1}^{0} & \ldots & \Sigma_{2 n}^{0}
\end{array}\right)
\end{aligned}
$$

Let $D$ be $\operatorname{det}\left(M^{T} M\right)$, applying the Cramer's rule gives:

$$
A_{j, k-j}=\operatorname{det}\left(\begin{array}{ccccc}
\Sigma_{0}^{0} & \ldots & B_{0,0} \Sigma_{0}^{0}+B_{1,0} \Sigma_{0}^{1}+B_{0,1} \Sigma_{1}^{0}+\ldots+B_{0, n} \Sigma_{n}^{0}+O\left(h^{n+1}\right) & \ldots & \Sigma_{n}^{0} \\
\Sigma_{0}^{1} & \ldots & B_{0,0} \Sigma_{0}^{1}+\ldots+B_{0, n} \Sigma_{n}^{1}+O\left(h^{n+2}\right) & \ldots & \Sigma_{n}^{1} \\
\Sigma_{1}^{0} & \ldots & B_{0,0} \Sigma_{1}^{0}+\ldots+O\left(h^{n+2}\right) & \ldots & \Sigma_{n+1}^{0} \\
\vdots & \vdots & \vdots & \vdots & \vdots \\
\Sigma_{n}^{0} & \ldots & B_{0,0} \Sigma_{n}^{0}+\ldots+O\left(h^{n+n+1}\right) & \ldots & \Sigma_{2 n}^{0}
\end{array}\right) / D
$$

Linear combinations of the columns gives:

$$
A_{j, k-j}=\operatorname{det}\left(\begin{array}{ccccc}
\Sigma_{0}^{0} & \ldots & B_{j, k-j} \Sigma_{k-j}^{j}+O\left(h^{n+1}\right) & \ldots & \Sigma_{n}^{0} \\
\Sigma_{0}^{1} & \ldots & B_{j, k-j} \Sigma_{k-j}^{j+1}+O\left(h^{n+2}\right) & \ldots & \Sigma_{n}^{1} \\
\Sigma_{1}^{0} & \ldots & B_{j, k-j} \Sigma_{k-j+1}^{j}+O\left(h^{n+2}\right) & \ldots & \Sigma_{n+1}^{0} \\
\vdots & \vdots & \vdots & \vdots & \vdots \\
\Sigma_{n}^{0} & \ldots & B_{j, k-j} \Sigma_{k-j+n}^{j}+O\left(h^{n+n+1}\right) & \ldots & \Sigma_{2 n}^{0}
\end{array}\right) / D
$$

The numerator of this formula splits due to the multi-linearity of the determinant, and noticing that $\Sigma_{l}^{k}=$ $O\left(h^{k+l}\right)$ gives $A_{j, k-j}=B_{j, k-j}+O\left(h^{n-k+1}\right)$. 
With respect to the order of convergence, it is equivalent to performing the fitting in any coordinate system. Nevertheless, as noted above, the error estimates are better if the convex hull of the sample points is not too flat. Consequently, it means that for the best estimates one should take a coordinate system as close as possible to the Monge system. Using the previous proposition, the order of accuracy of a differential quantity is easily related to the degree of the interpolant and the order of this quantity. More precisely:

Theorem. 3 A polynomial fitting of degree $n$ estimates any $k^{\text {th }}$-order differential quantity to accuracy $O\left(h^{n-k+1}\right)$. In particular:

- the coefficients of the first fundamental form and the unit normal vector are estimated with accuracy $O\left(h^{n}\right)$, and so is the angle between the normal and the estimated normal.

- the coefficients of the second fundamental form and the shape operator are approximated with accuracy $O\left(h^{n-1}\right)$, and so are the principal curvatures and directions (as long as they are well defined, i.e. away from umbilics).

Proof.It is easily checked that the formula corresponding to the geometric quantities (as long as they are well defined) are $C^{1}$ functions of the coefficients. The result follows from proposition 1 and lemma 1 - see appendix in section 9 .

The previous theorem generalizes [MW00, Lemma 4.1] where 2-jet interpolations only are studied. The $O\left(h^{n}\right)$ bound on the normal should also be compared to the estimate of the normal vector using specific Voronoi centers called poles considered in [AB99]. The error bound proved there is equivalent to $2 \varepsilon$ with $\varepsilon$ the sampling density of the surface. Letting lfs stand for local feature size, setting $h=\varepsilon$ lfs and assuming lfs is bounded from above, the estimation stemming from a polynomial fitting therefore yields more accurate results for the tangent plane, and also provides information on higher order quantities.

\subsection{Influence of normal accuracy on higher order estimates}

Following the guideline initiated in [SZ90] several algorithms first estimate the normal to the surface and then proceed with Hessian of the height function. We analyze the error incurred by the latter as a function of the accuracy on the former. We denote $\theta$ the angle between the normal $n_{S}$ to the surface and the normal $n_{Q}$ estimated by the twostages method. In order to simplify calculations, we assume that $n_{Q}$ is aligned with the $z$-axis and $n_{S}$ is in the $(x, z)$ plane, so that $f(x, y)=B_{10} x+B_{20} x^{2}+B_{11} x y+B_{02} y^{2}+O\left(\|(x, y)\|^{3}\right)$, with $B_{10}=-\tan \theta$. Expressed in the same coordinate system, the interpolant - a 2-jet to simplify calculations- reads as $J_{A, 2}(x, y)=A_{20} x^{2}+A_{11} x y+A_{02} y^{2}$.

Proposition. 2 If a small error $\theta$ is done on the estimated normal, a 2-jet interpolation give the Gauss curvature with a linear error wrt $\theta$ :

$$
k_{Q}-k_{S}=\theta O\left(h^{-1}\right)+O(h)+O\left(\theta^{2}\right) .
$$

Proof. The system of equations for the interpolation is:

$$
A_{2,0} x_{i}^{2} h+A_{1,1} x_{i} y_{i}+A_{0,2} y_{i}^{2}=B_{1,0} x_{i}+B_{2,0} x_{i}^{2}+B_{1,1} x_{i} y_{i}+B_{0,2} y_{i}^{2}+O\left(\left\|\left(x_{i}, y_{i}\right)\right\|^{3}\right) \quad i=1, \ldots, 3 .
$$

Let $D$ be the determinant: $D=\operatorname{det}\left(x_{i}^{2}, x_{i} y_{i}, y_{i}^{2}\right)_{i=1, \ldots, 3}$, Cramer's rule gives:

$$
\begin{aligned}
A_{2,0} & =\operatorname{det}\left(B_{1,0} x_{i}+B_{2,0} x_{i}^{2}+B_{1,1} x_{i} y_{i}+B_{0,2} y_{i}^{2}+O\left(\left\|\left(x_{i}, y_{i}\right)\right\|^{3}\right), x_{i} y_{i}, y_{i}^{2}\right)_{i=1, \ldots, 3} / D \\
& =B_{1,0} O\left(h^{-1}\right)+B_{2,0}+O(h) .
\end{aligned}
$$

Similar calculations gives: $A_{1,1}=B_{1,0} O\left(h^{-1}\right)+B_{1,1}+O(h)$, and $A_{0,2}=B_{1,0} O\left(h^{-1}\right)+B_{0,2}+O(h)$. The Gaussian curvature of $Q$ is then:

$$
\begin{aligned}
k_{Q} & =\frac{4 A_{2,0} A_{0,2}-A_{1,1}^{2}}{1+A_{1,0}^{2}} \\
& =4 A_{2,0} A_{0,2}-A_{1,1}^{2} \\
& =4\left(B_{1,0} O\left(h^{-1}\right)+B_{2,0}+O(h)\right)\left(B_{1,0} O\left(h^{-1}\right)+B_{0,2}+O(h)\right)-\left(B_{1,0} O\left(h^{-1}\right)+B_{1,1}+O(h)\right)^{2} \\
& =4 B_{2,0} B_{0,2}-B_{1,1}^{2}+B_{1,0} O\left(h^{-1}\right)+B_{1,0}^{2} O\left(h^{-2}\right)+O(h) \\
& =4 B_{2,0} B_{0,2}-B_{1,1}^{2}+\tan \theta O\left(h^{-1}\right)+\tan ^{2} \theta O\left(h^{-2}\right)+O(h) .
\end{aligned}
$$


The Gaussian curvature of $S$ is

$$
k_{S}=\frac{4 B_{2,0} B_{0,2}-B_{1,1}^{2}}{\left(1+B_{1,0}^{2}\right)^{2}}=\frac{4 B_{2,0} B_{0,2}-B_{1,1}^{2}}{\left(1+\tan ^{2} \theta\right)^{2}}=\left(4 B_{2,0} B_{0,2}-B_{1,1}^{2}\right) \cos ^{4} \theta .
$$

Thus the error on the curvature is:

$$
\begin{aligned}
k_{Q}-k_{S} & =\left(4 B_{2,0} B_{0,2}-B_{1,1}^{2}\right) \cos ^{4} \theta+\tan \theta O\left(h^{-1}\right)+\tan ^{2} \theta O\left(h^{-2}\right)+O(h) \\
& =\theta O\left(h^{-1}\right)+O(h)+O\left(\theta^{2}\right) .
\end{aligned}
$$

For a fixed $h$, the curvature error is a linear function of the angle between the normals. The term $\theta O\left(h^{-1}\right)$ shows that if $\theta$ is fixed, the smaller $h$ the worse the accuracy. Hence estimating the normal deserves specific care.

\section{Plane Curves}

All the results proved for surfaces in the previous section can also be proved for curve, and we omit them. Instead, for the interpolation case, we prove an error bound between the coefficients of the curve and those of the jet.

\subsection{Problem addressed}

Let $C$ be a curve, and consider the height function $f$ following Equation (1) in any coordinate system whose $y$ axis is not tangent to the curve and, whose origin is on the curve (this implies that $B_{0}=0$ ). We shall fit $C$ by a $n$-jet $J_{A, n}(x)$. As already mentioned, there are $n+1$ unknown coefficients $A_{i}$, we assume $N$ data points $P_{i}\left(x_{i}=a_{i} h, y_{i}=f\left(x_{i}\right)\right)$ are given, where $N=n+1$ for interpolation fitting. Notice again that parameter $h$ specifies the uniform convergence of these data points to the origin. The fitting equations are:

$$
y_{i}=f\left(x_{i}\right)=J_{B, n}\left(x_{i}\right)+O\left(x_{i}^{n+1}\right)=J_{A, n}\left(x_{i}\right) .
$$

Since curve $C$ is given by Equation (1), the normal and the curvature of $C$ at the origin are given by

$$
n_{C}=\left(-B_{1}, 1\right)^{t} / \sqrt{1+B_{1}^{2}}, k_{C}=2 B_{2} /\left(1+B_{1}^{2}\right)^{\frac{3}{2}} .
$$

Moreover, in the Monge coordinate system $-B_{1}=0$, these expressions simplify to $n_{C}=(0,1)^{t}$ and $k_{C}=2 B_{2}$.

\subsection{Error bounds for the interpolation}

The equivalent of Prop. 1 for curves gives the magnitude of the accuracy of the interpolation. We can actually be more precise and provide error bounds depending upon the function interpolated and the position of the interpolation points.

Proposition. 3 Consider a degree $n(n \geq 1)$ interpolation problem for a curve $y=f(x)$. Let $h$ be a positive number so that the interpolation point abscissa lie in the interval $[-h, h]$, and $c=\sup _{x \in[-h, h]}\left|f^{(n+1)}(x)\right|$. Then for $k=0, \ldots, n$ :

$$
\left|A_{k}-B_{k}\right| \leq \frac{h^{n-k+1} c}{k !(n-k+1) !} .
$$

Proof. This result is a simple application of the analysis of the Lagrange interpolation remainder which can be found in [EK66]. Let $R_{n}(x)=f(x)-J_{A}(x)$, theorem 1 p.289 states that $\forall k=0, \ldots, n$ and $\forall x \in[-h, h]$ :

$$
R_{n}^{(k)}(x)=\prod_{j=0}^{n-k}\left(x-\xi_{j}\right) \frac{f^{(n+1)}(\eta)}{(n+1-k) !}
$$


with $x, \xi_{j}, \eta \in[-h, h]$.

For $x=0$, this leads to:

$$
\begin{aligned}
& \left|R_{n}^{(k)}(0)\right|=\left|k ! A_{k}-k ! B_{k}\right| \leq \frac{h^{n-k+1} c}{(n-k+1) !} \\
& \left|A_{k}-B_{k}\right| \leq \frac{h^{n-k+1} c}{k !(n-k+1) !}
\end{aligned}
$$

Here is an application of the previous result. Let $\theta$ denote the angle between the normal and the estimated normal. We have $\sin (\theta)=\left\|n_{Q} \wedge n_{C}\right\|=\left|A_{1}-B_{1}\right| / \sqrt{\left(1+A_{1}^{2}\right)\left(1+B_{1}^{2}\right)} \leq\left|A_{1}-B_{1}\right|$. It is found that

$$
\theta \leq \arcsin \left(\frac{h^{n} c}{n !}\right) \text {. }
$$

\section{Algorithm}

The fitting algorithm to estimate the differential properties at a point $p$ consists of (i)collecting the $N$ points used for the fitting. (Recall that a $n$-jet involves $N_{n}=(n+1)(n+2) / 2$ coefficients, so that when interpolating (approximating) we assume $N=N_{n}\left(N>N_{n}\right)$.) (ii)solving the linear system (iii)recovering the differential properties.

We examine in turn the details of these three steps.

\subsection{Collecting $N$ neighbors}

The mesh case. Although no topological information is required by the fitting method, the connectivity information of a mesh can be used as follows. We sequentially visit the one-ring neighbors, two-ring neighbors, and so on until $N$ points have been collected. Let $R_{1}, \ldots, R_{k}$ be the $k$ rings of neighbors necessary to collect $N$ neighbors. All the points of the $k-1$ first rings are used. The complement up to $N$ points is chosen arbitrarily out of the $k$ th ring.

The point-cloud case. The normal at $p$ is first estimated, and the neighbors of $p$ are further retrieved from a power diagram in the estimated tangent plane [BF02] — a provably good procedure if the samples are dense enough. If the number of neighbors collected is less than $N$, we recursively collect the neighbors of the neighbors.

Collecting the points therefore boils down to estimating the tangent plane. One solution is to construct the Voronoi diagram of the point set and use these Voronoi vertices called poles [AB99]. Poles yield an accurate estimate of the normal vector but require a global construction.

An alternative is to resort to the algorithm of section 4, and solve a degree one interpolation problem — which requires three points and is well poised as soon as the three points are not collinear. Geometrically, the closer the three points to being aligned, the more unstable the tangent plane estimate. To see how one can get around this difficulty, denote $q$ the nearest neighbor of $p$. Also, let $r$ be the sample point so that the circum-radius $r_{\text {circ }}$ of the triangle $p q r$ is minimum. The estimated normal at $p$ is the normal to the plane through $p q r$. Intuitively, minimizing the circum-radius $r_{\text {circ }}$ prevents two difficulties: on one hand triangles with a large angle (near to $\pi$ ) exhibit a large circum-circle and are discarded; on the other hand, triangles involving a third point $r$ which is not a local neighbor of $p$ cannot minimize $r_{c i r c}$ and are also discarded. A more formal argument advocating the choice of the triangle with minimum $r_{\text {circ }}$ is provided in [She02], where it is shown that the worst error on the approximation of the gradient of a bivariate function by a linear interpolant precisely involves $r_{\text {circ }}$.

\subsection{Solving the fitting problem}

The next stage consists of choosing the $z$ direction to work with. Since the tangent plane has not been estimated, we use a principal component analysis to compute a rough estimate of the normal with the collected points. The polynomial fitting can be done in any coordinate system whose $z$ axis is not tangent to the surface. Hence at least one of the three axis of the world coordinate system matches this requirement. A natural choice is to select the coordinate axis whose angle with the rough estimated normal is minimum. Another choice is that of a coordinate system for which the $z$ axis is the rough estimated normal. This choice may increase the ratio $d_{\max } / d_{\min }$ and improve 
the results as explained in section 4, but this requires the calculation of a non trivial coordinate transformation. The improvement of this latter choice has not been studied experimentally since the first alternative performs well enough to confirm the theoretical orders of convergence — cf. section 7.1. For the chosen coordinates, we fill the Vandermonde matrix. The matrix is further pre-conditioned as explained in section 3.3, with $h$ the average value of the norms $\left\|\left(x_{i}, y_{i}\right)\right\|$. The corresponding system is solved using a Singular Value Decomposition. Practically, we use the SVD of the Gnu Scientific Library, available from http://sources.redhat.com/gsl.

As pointed out in section 3.3, the instability of the system is provided by the condition number. Whenever degenerate configurations are detected, one can proceed as follows. For the approximation strategy, one can either keep the same degree and increase the number of points used, or reuse the same points with a lower degree. These changes are likely to provide a non singular matrix $M$. In the worst-case, a degree one fitting must be possible since then only three linearly independent points are required! For the interpolation, things are a bit more involved since reducing the interpolation degree requires discarding some points. Selecting the subset yielding the best conditioning is a challenging problem [Las99, Hig96]. Notice also that for the approximation case, one can always retrieve a solution from an under-constrained least-square problem by choosing, e.g., the solution vector of least norm.

\subsection{Retrieving differential quantities}

We have already mentioned how to compute the normal. For the second order information, we compute the Weingarten map of the surface [dC76, Section 3.3]. Its eigenvalues (eigenvectors) provide the principal curvatures (directions) of the surface. For a parameterized surface given as a height function, one ends up with the formula given on Table 1. Notice that a basis of the tangent space associated to the parameterization $X(u, v)=(u, v, h(u, v))$ consists of the two vectors $X_{u}=\left(1,0, h_{u}\right)^{t}$ and $X_{v}=\left(0,1, h_{v}\right)^{t}$. A Gram-Schmidt orthonormalization of the basis $\left\{X_{u}, X_{v}\right\}$ gives another basis $\{Y, Z\}$ of the tangent space. The diagonalization of the symmetric matrix representing the Weingarten map in the basis $\{Y, Z\}$ provides the expression of the principal curvature directions with respect to the $\{Y, Z\}$ orthonormal basis. Note that the sign of principal curvatures and hence the definition of minimal and maximal directions rely on the orientation of the normal. As long as our experimental study is performed on meshes of oriented surfaces, it is straightforward to find a global and coherent orientation of the normals.

$$
\begin{array}{lll}
E=1+a_{1}{ }^{2} & e=\frac{2 a_{3}}{\sqrt{a_{1}^{2}+1+a_{2}{ }^{2}}} & \\
F=a_{2} a_{1} & f=\frac{a_{4}}{\sqrt{a_{1}^{2}+1+a_{2}^{2}}} & A^{t}=-\left(\begin{array}{ll}
e & f \\
f & g
\end{array}\right)\left(\begin{array}{ll}
E & F \\
F & G
\end{array}\right)^{-1} \\
G=1+a_{2}{ }^{2} & g & =\frac{2 a_{5}}{\sqrt{a^{2}+1+a^{2}}}
\end{array}
$$

Table 1: Computing the matrix $A$ of the Weingarten map of $h(u, v)=a_{1} u+a_{2} v+a_{3} u^{2}+a_{4} u v+a_{5} v^{2}$ in the basis $\left\{X_{u}, X_{v}\right\}$

\section{Experimental study}

We present results along two lines. First, we illustrate the convergence theorems proved in section 4. Second, we present illustrations on standard computer graphics models.

\subsection{Convergence estimates on a graph}

Setup. We illustrate the convergence properties with the smooth height fields $f(u, v)=0.1 e^{2 u+v-v^{2}}$ and $g(u, v)=$ $4 u^{2}+2 v^{2}$ defined over the parametric domain $(u, v) \in[-0.5,0.5]^{2}$ - see Figs. 4 and 5. At selected points on the graphs of $f$ and $g$, we study the angle between the normals - more precisely its $\operatorname{sine} \sin (n, \tilde{n})$, and the relative errors on principal curvatures. The values output by our algorithm are compared against the exact values computed analytically with arbitrary precision under Maple, and we report both average and maximum errors over sample points. More precisely, the graph of $f$ or $g$ are sampled with points $p_{i}\left(x_{i}, y_{i}, f\left(x_{i}, y_{i}\right)\right)$ where the $\left(x_{i}, y_{i}\right)$ lies on a randomly perturbed triangulated square grid of side $h$. The triangulation is randomly perturbed to avoid simple degenerate configurations such as points lying on lines. The perturbation for the point $(u, v)$ of the regular grid is the point $(x, y)$ with $x=u+\delta h, y=v+\delta^{\prime} h$ and $\delta, \delta^{\prime}$ random numbers in $[0,0.9]$. The connectivity of the graph is that of the triangulated grid. Notice also that since calculations are carried out on a surface patch parameterized 
over a square grid, the direction chosen for the polynomial fitting is the $z$ direction near the origin, and either the the $x$ or $y$ directions at the periphery of the domain.

The convergence properties are illustrated (i)with respect to the discretization step $h$ of the grid - for a given fitting degree $n$ (ii)with respect to the fitting degree $n$-for a given discretization step $h$. We compare the convergence properties of the interpolation and approximation schemes, for fitting degrees ranging from one to nine. To quantify the observations, notice that according to theorem 3 , the error $\delta$ on a $k^{\text {th }}$-order differential quantity is $O\left(h^{n-k+1}\right)$, hence

$$
\begin{aligned}
\delta \approx c h^{n-k+1} & \Leftrightarrow \log (1 / \delta) \approx \log (1 / c)+(n-k+1) \log (1 / h) \\
& \Leftrightarrow \frac{\log (1 / \delta)}{\log (1 / h)} \approx \frac{\log (1 / c)}{\log (1 / h)}+(n-k+1) .
\end{aligned}
$$

Convergence wrt to $h$. To highlight the convergence properties with respect to the size of the neighborhood, we consider sequences of meshes with $h \rightarrow 0$, more precisely $h$ ranges from $2^{-2}$ to $2^{-6}$. The results for $f$ and $g$ being alike, we focus on the exponential $f$.

Curves of Figs. 6 to 11 show the average convergence behavior as the size of the neighborhood decreases. For a given degree and following equation (15), curves of figures 6 to 11 should be lines of slope $(n-k+1)$. The behavior is more regular for the approximation case, and the estimate is also better: a gain of about a digit can be observed between $k_{\max }$ estimates of figures 10 and 11 .

Convergence wrt to the interpolation degree. For the convergence wrt to the interpolation degree -with $h$ fixed, we present results for the polynomial $g$ (cf. Fig. 12 to 17). Conclusions are similar for $f$ (cf. Fig. 24 to 29), but it should however be noticed that since the graph of $g$ is more curvy than that of $f$, a finer grid is required. (The higher the degree the more points required by the fitting ... and we compute local quantities!) To be precise, we ran experiments with $h=2^{-5}$ for $f$ and $h=2^{-7}$ for $g$.

Curves of figures 12 and 13 show the convergence as a function of the degree of the fitted polynomial for a fixed neighborhood size. According to Eq. (16), curves of these figures should be lines of unit slope, with a vertical shift of one unit between normal and curvatures errors since curvature is a $2^{\text {nd }}$ order quantity whereas normal is $1^{s t}$ order. The gap between the average values and the maximal values is greater for interpolation than for approximation. The other charts provide the conditioning and the least singular value. Interpolation fitting is always more ill-conditioned than approximation, and closer to a degenerate problem (the least singular value is the distance of the matrix system to singular matrices). The particular case of a degree 7 approximation reveals to be badly conditioned due to the regular connectivity of the mesh used to find the neighbors: there is only one more point than for the degree 7 interpolation fitting.

\subsection{Illustrations}

We depict differential informations on several models. When principal directions are displayed, blue and red respectively correspond to $k_{\min }$ and $k_{\max }$ - that is $k_{\min } \leq k_{\max }$ 一, assuming the surface normal points to the outside. To display patches of osculating $n$-jets, it is sufficient to select a rectangular domain in parameter space, sample it with a grid, and plot the corresponding mesh.

Consider the mesh models of the elliptic paraboloid $z=2 x^{2}+y^{2}-16 \mathrm{k}$ points, Fig. 18-, and the surface of revolution $\left.z=0.1 \sin \left(10 \sqrt{(} x^{2}+y^{2}\right)\right)-8 \mathrm{k}$ points, Fig. 19. The arrangement of curvature lines provides informations on umbilical points - where principal directions are not defined since $k_{\min }=k_{\max }$. On the paraboloid, it is easy to follow curvature lines and see how they turn around an umbilic. The surface of revolution provides an example of two parabolic lines (where the principal curvature $k_{\max }$ vanishes), that is a curve along which the Gauss curvature $K_{\text {Gauss }}$ vanishes. This specific line splits the surface into elliptic $\left(K_{\text {Gauss }}>0\right)$ and hyperbolic regions $\left(K_{\text {Gauss }}<0\right)$. This model also illustrates a line of umbilical points where minimum and maximum principal directions swap each over.

For a standard example from Computer Graphics, consider the Michelangelo's David of Fig. 20. On this model of 95922 pts, the principal curvatures provide meaningful information for shape perception ${ }^{5}$. To finish up, we illustrate the robustness of the method. Figure 21 displays random patches on the Mechanic model, a 12,500 points model reconstructed from the output of a range scanner. In spite of the coarse sampling, patches and

${ }^{5}$ See also [HCV52, p197] as well as [HGY $\left.{ }^{+} 99\right]$. 
principal directions provide faithful information. In a similar vein, approximation fitting with large neighborhoods Fig. 22 features a noisy triangulation of a graph. In spite of the severe level of noise, surface patches average the available information. On Fig. 23, a noisy triangulation of an ellipsoid, 15k points, principal directions are enough precise to recognize an umbilic.

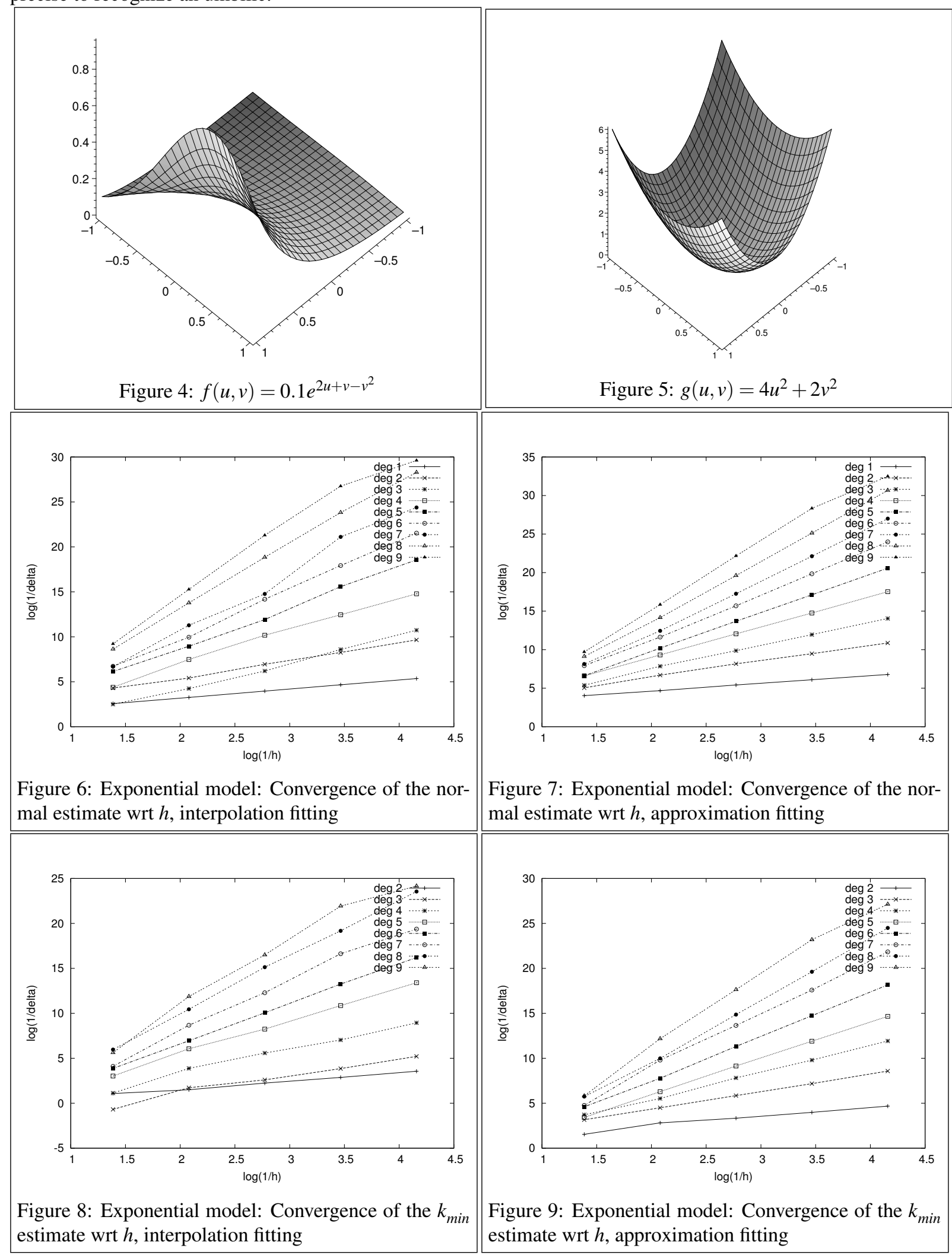




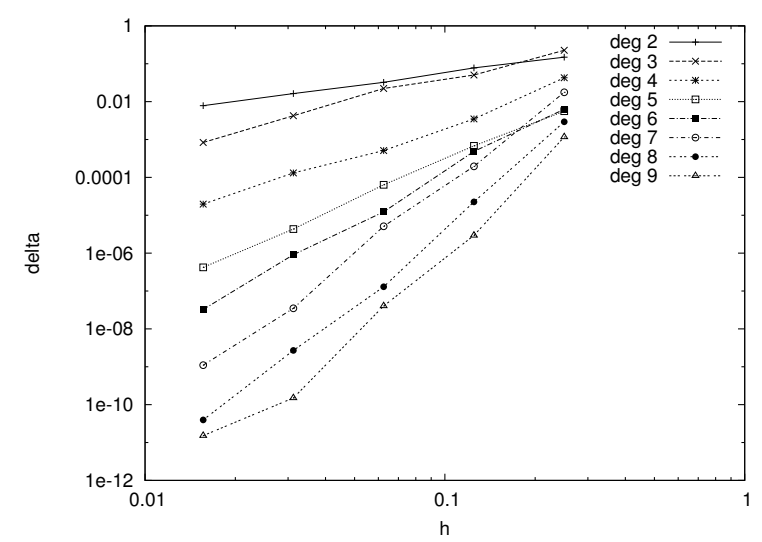

Figure 10: Exponential model: Convergence of the $k_{\max }$ estimate wrt $h$, interpolation fitting

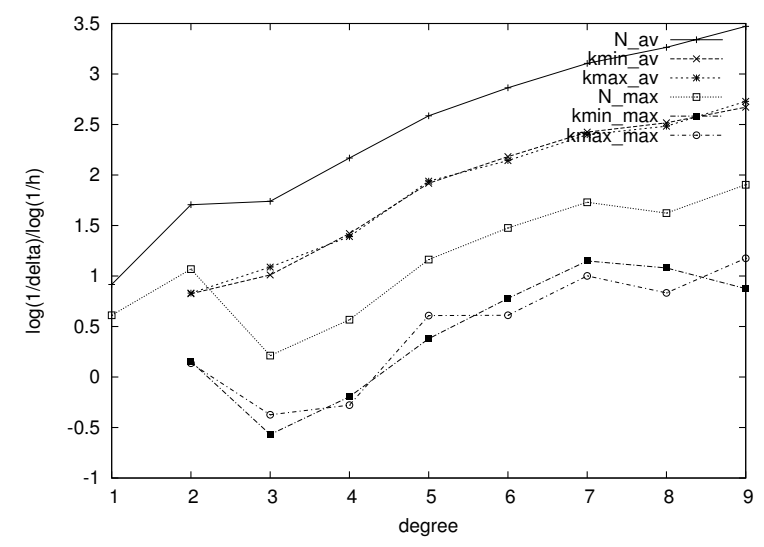

Figure 12: Polynomial model: Convergence of normal and curvature estimates wrt the degree of the interpolation fitting

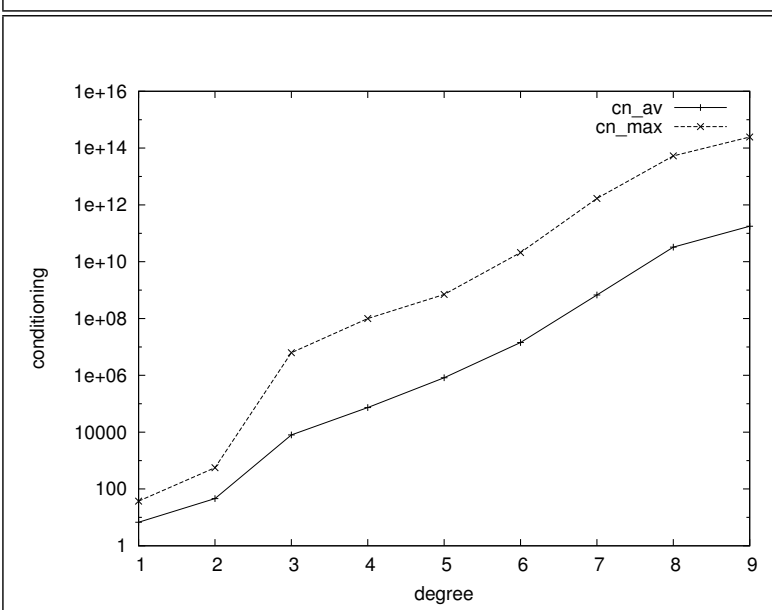

Figure 14: Polynomial model: Conditioning wrt the degree of the interpolation fitting

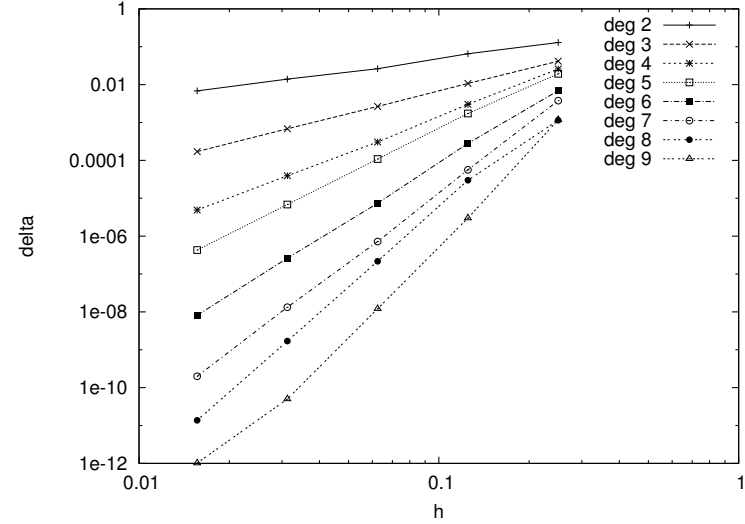

Figure 11: Exponential model: Convergence of the $k_{\max }$ estimate wrt $h$, approximation fitting

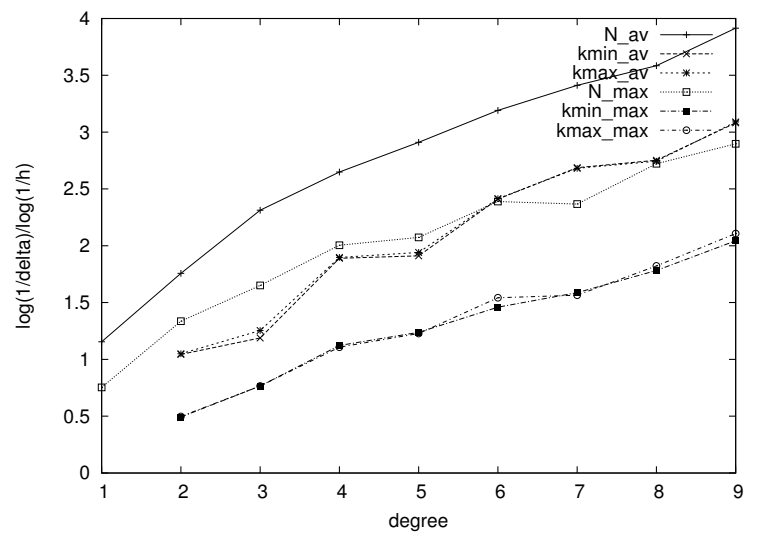

Figure 13: Polynomial model: Convergence of normal and curvature estimates wrt the degree of the approximation fitting

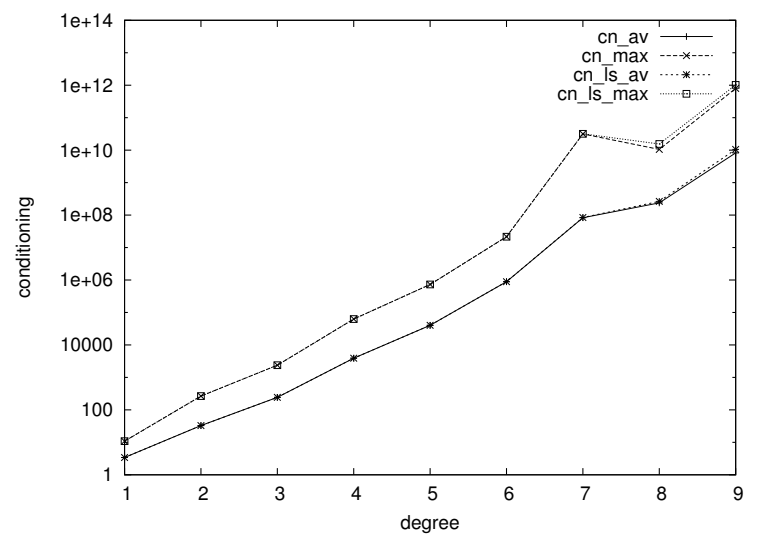

Figure 15: Polynomial model: Conditioning wrt the degree of the approximation fitting 


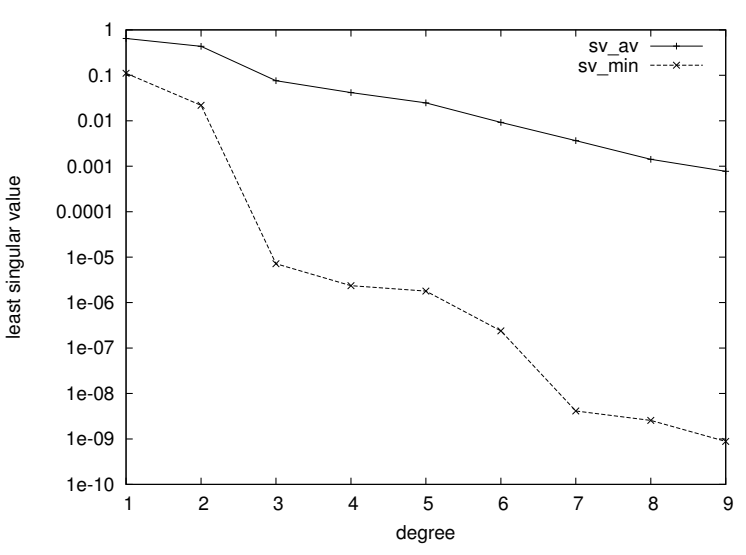

Figure 16: Polynomial model: Least singular value wrt the degree of the interpolation fitting

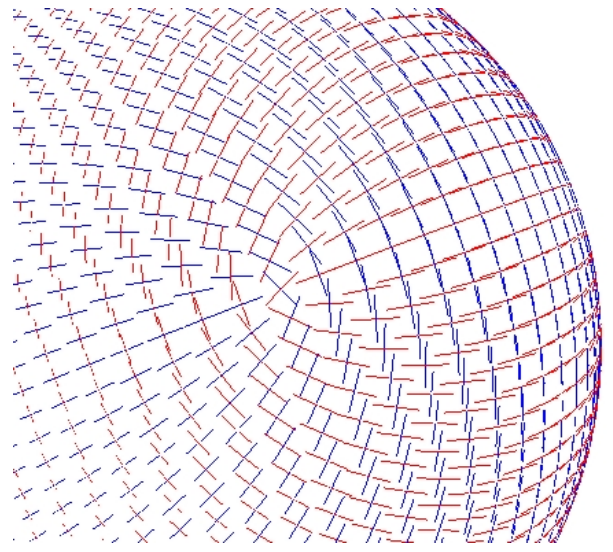

Figure 18: Elliptic paraboloid

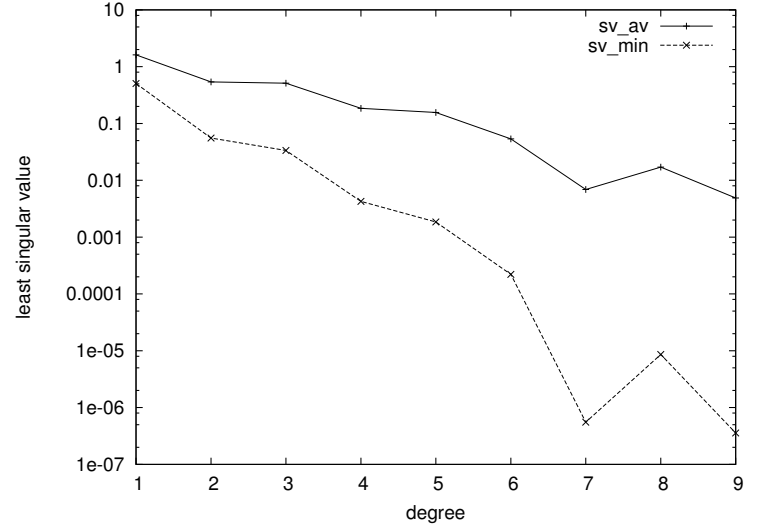

Figure 17: Polynomial model: Least singular value wrt the degree of the approximation fitting

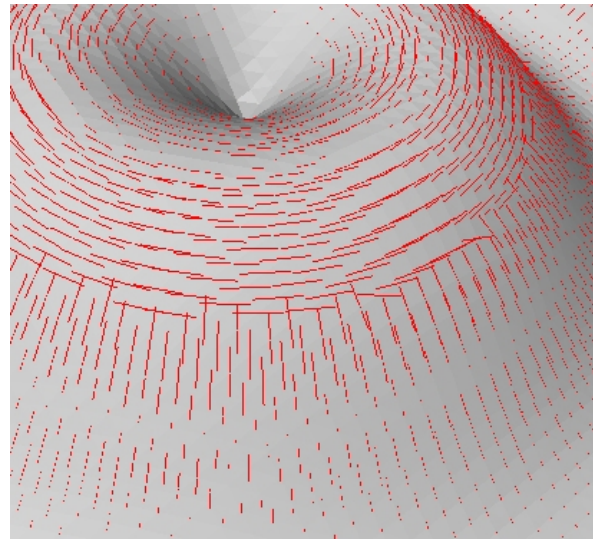

Figure 19: Surface of revolution 


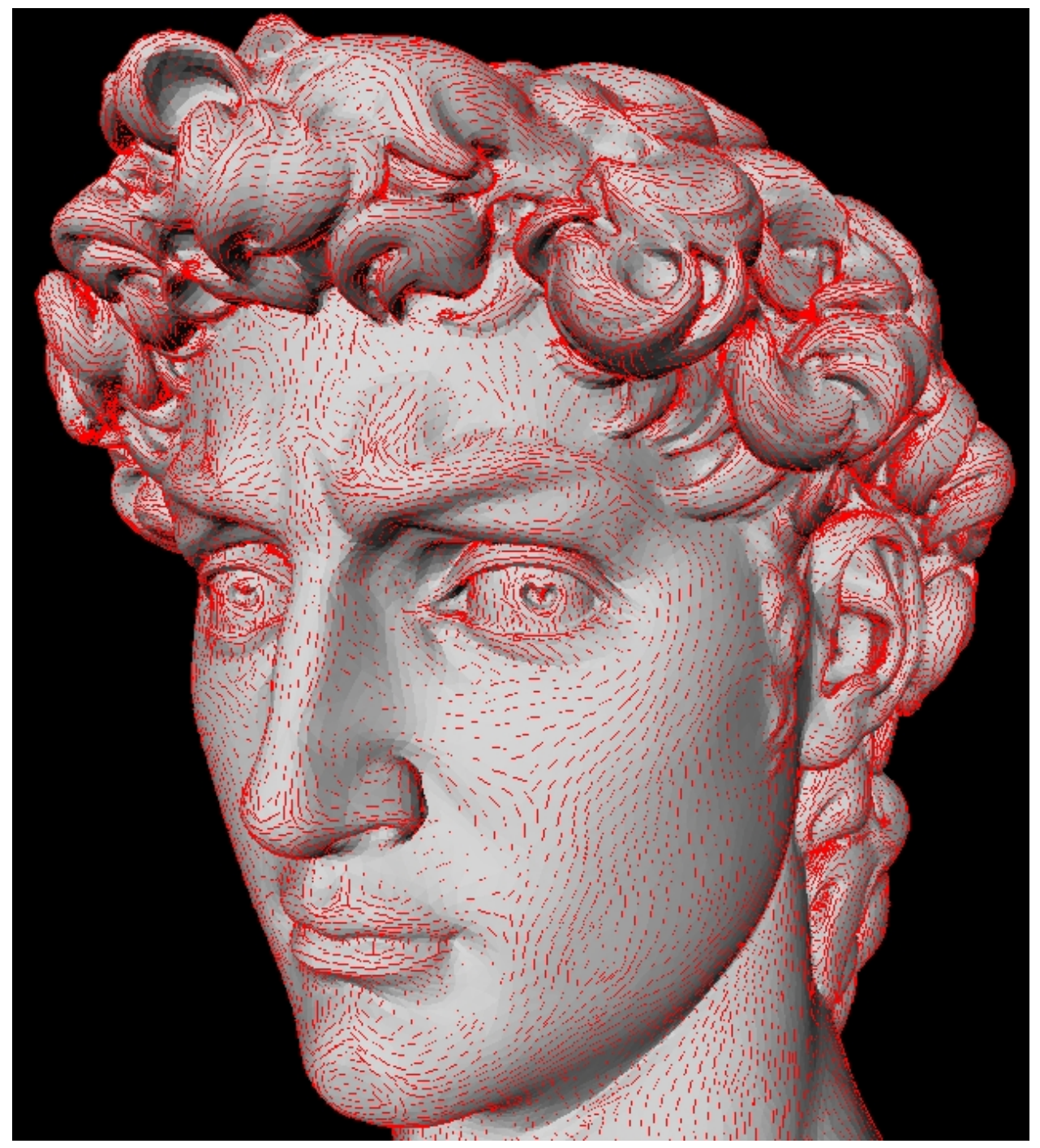

Figure 20: Michelangelo's David: principal directions associated with $k_{\max }$ scaled by $k_{\min }$

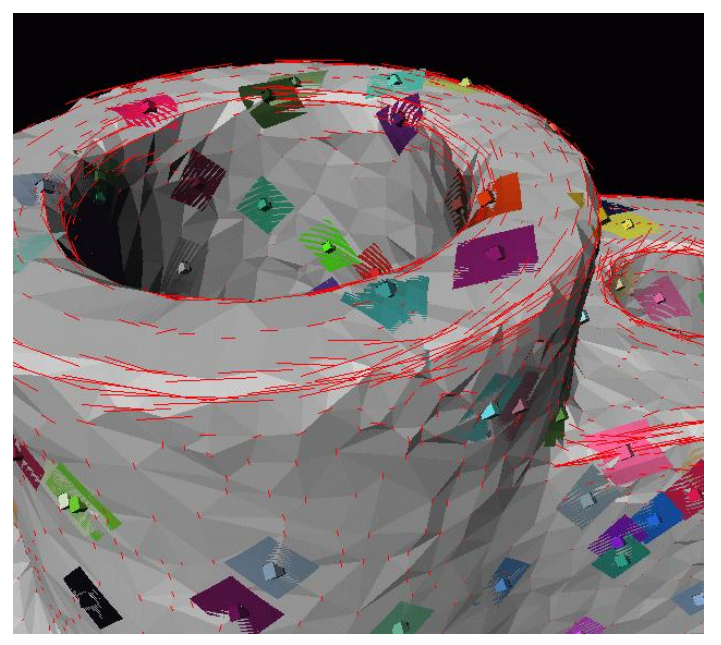

Figure 21: Mechanic: closeup 


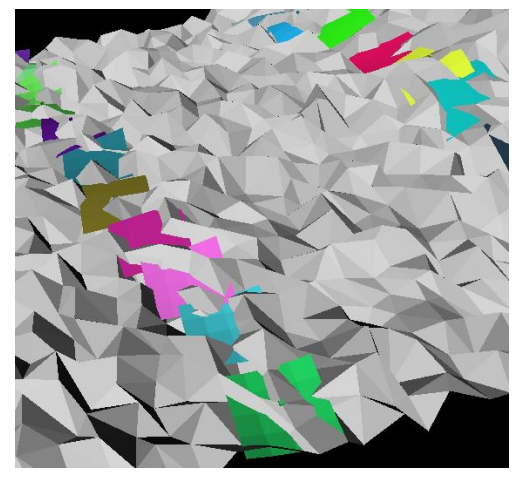

Figure 22: $f(u, v)=u+3 v+e^{2 u+v-v^{2}}$ with noise

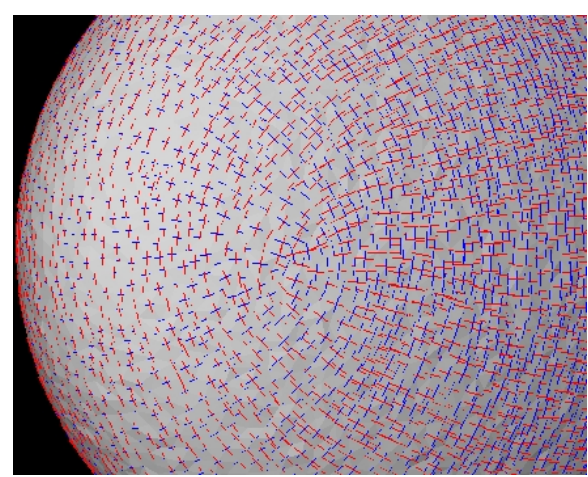

Figure 23: Principal directions on a noisy ellipsoid

\section{Conclusion}

Estimating differential quantities is of prime importance in many applications from Computer Vision, Computer Graphics, Computer Aided Design or Computational Geometry. This importance accounts for the many different differential estimators one can find in the vast literature of applied geometry. Unfortunately, few of these have undergone a precise theoretical analysis. Another striking fact is that estimates of second order differential quantities are always computed using degenerate conics/quadrics without even mentioning the classification of Euclidean conics/quadrics.

The main contribution of the paper is to bridge the gap between the question of estimating differential properties of arbitrary order and multivariate interpolation and approximation. In making this connection, the use of jets - truncated Taylor expansions - is advocated. Precise asymptotic convergence rates are proved for curves and surfaces, both for the interpolation and approximation schemes. To the best of our knowledge, these results are among the first ones providing accurate estimates for differential quantities of order three and more. Experimental results for surfaces of $\mathbb{R}^{3}$ are reported. These experiments illustrate the asymptotic convergence results, but also the robustness of the methods on general Computer Graphics models.

Acknowledgments. The authors wish to thank P. Chenin for pointing out Ref. [EK66], and the reviewers for providing an indirect link to Ref. [QV94].

\section{References}

[AB99] Nina Amenta and Marshall Bern. Surface reconstruction by Voronoi filtering. Discrete Comput. Geom., 22(4):481-504, 1999.

[BCM02] V. Borrelli, F. Cazals, and J-M. Morvan. On the angular defect of triangulations and the pointwise approximation of curvatures. In Curves and Surfaces, St Malo, France, 2002. INRIA Research Report RR-4590.

[Ber87] M. Berger. Geometry (vols. 1-2). Springer-Verlag, 1987.

[BF02] J.-D. Boissonnat and J. Flototto. A local coordinate system on a surface. In ACM symposium on Solid modeling, Saarbrücken, 2002.

[BG92] J.W. Bruce and P.J. Giblin. Curves and singularities (2nd Ed.). Cambridge, 1992.

[Coa66] C. Coatmelec. Approximation et interpolation des fonctions differentielles de plusieurs variables. Ann. Sci. Ecole Norm. Sup., 83, 1966.

[CP04] F. Cazals and M. Pouget. Smooth surfaces, umbilics, lines of curvatures, foliations, ridges and the medial axi: a concise overview. Technical Report 5138, INRIA, 2004.

[CR72] P.G. Ciarlet and P.-A. Raviart. General lagrange and hermit interpolation in $r^{n}$ with applications to the finite element method. Arch. Rational Mesh. Engrg., 46:177-199, 1972. 
[CSM03] D. Cohen-Steiner and J.-M. Morvan. Restricted delaunay triangulations and normal cycle. In $A C M$ Symposium on Computational Geometry, 2003.

[CW00] P. Csàkàny and A.M. Wallace. Computation of local differential parameters on irregular meshes. In Springer, editor, Mathematics of Surfaces. R. Cipolla and R. Martin Eds, 2000.

[dC76] M. do Carmo. Differential Geometry of Curves and Surfaces. Prentice-Hall, 1976.

[EK66] E.Isaacson and H.B. Keller. Analysis of numerical methods. John Wiley \& Sons, 1966.

[GI04] J. Goldfeather and V. Interrante. A novel cubic-order algorithm for approximating principal direction vectors. ACM Trans. Graph., 23(1):45-63, 2004.

[GS00] M. Gasca and T. Sauer. Polynomial interpolation in several variables. Advances in Comp. Math., 12(4), 2000.

[GvL83] G. Golub and C. van Loan. Matrix Computations. Johns Hopkins Univ. Press, Baltimore, MA, 1983.

[HCV52] D. Hilbert and S. Cohn-Vossen. Geometry and the Imagination. Chelsea, 1952.

$\left[\mathrm{HGY}^{+} 99\right]$ P. W. Hallinan, G. Gordon, A.L. Yuille, P. Giblin, and D. Mumford. Two-and Three-Dimensional Patterns of the Face. A.K.Peters, 1999.

[Hig96] N.J. Higham. Accuracy and Stability of Numerical Algorithms. SIAM, 1996.

[Las99] M. Lassak. Parallelotopes of maximum volumes in a simplex. Disc. Comp. Geometry, $21,1999$.

[LS86] P. Lancaster and K. Salkauskas. Curve and surface fitting: an introduction. Academic, 1986.

[MT02] J-M. Morvan and B. Thibert. Smooth surface and triangular mesh : Comparison of the area, the normals and the unfolding. In ACM Symposium on Solid Modeling and Applications, 2002.

[MW00] D. Meek and D. Walton. On surface normal and Gaussian curvature approximations given data sampled from a smooth surface. Computer-Aided Geometric Design, 17:521-543, 2000.

[Pet01] S. Petitjean. A survey of methods for recovering quadrics in triangle meshes. ACM Computing Surveys, 34(2), 2001.

[Por01] I. Porteous. Geometric Differentiation (2nd Edition). Cambridge University Press, 2001.

[PS98] K. Polthier and M. Schmies. Straightest geodesics on polyhedral surfaces. In Mathematical Visualization, H.C. Hege, K. Polthier Editors, 1998.

[QV94] A. Quarteroni and A. Valli. Numerical Approximation of Partial Differential Equations. Springer, 1994.

[Sau95] T. Sauer. Computational aspects of multivariate polynomial interpolation. Advances in Comp. Math., 3(3), 1995.

[She02] Jonathan R. Shewchuk. What is a good linear element? interpolation, conditioning, and quality measures. In 11th International Meshing Roundtable, Ithaca, New York, USA, 2002.

[Spi99] M. Spivak. A comprehensie introduction to Differential Geometry (Third Ed.). Publish or Perish, 1999.

[SX95] T. Sauer and Yuan Xu. On multivariate lagrange interpolation. Math. Comp., 64, 1995.

[SZ90] P. Sander and S. Zucker. Inferring surface trace and differential structure from 3-D images. IEEE Trans. Pattern Analysis and Machine Intelligence, 12(9):833-854, 1990.

[Tau95] G. Taubin. Estimating the tensor of curvature of a surface from a polyhedral approximation. In Fifth International Conference on Computer Vision, 1995.

[WB01] K. Watanabe and A.G. Belyaev. Detection of salient curvature features on polygonal surfaces. In Eurographics, 2001. 


\section{Appendix: a useful lemma}

In several occasions we derive differential quantities - unit normal vectors, curvatures - as a function $F$ of the coefficients of the jet. The following lemma makes it easy to derive the precision on the quantity investigated if $F$ is regular enough and if the some precision of the jet's coefficients is known.

Lemma. 1 Define a $k^{\text {th }}$-order differential quantity -for a curve or a surface- as a $C^{1}$ function $F$ of the of the coefficients of the $k$-jet of the height function. Also assume that a degree $n$ fitting yields a precision $A_{j}=$ $B_{j}+O\left(h^{n+1-j}\right), k=1, \ldots, n$ for a curve, and $A_{k-j, j}=B_{k-j, j}+O\left(h^{n-k+1}\right), j=0, \ldots, n, k=0, \ldots, n$ for a surface.

Then, a polynomial fitting of degree $n$ estimates a $k^{\text {th }}$-order differential quantity to accuracy $O\left(h^{n-k+1}\right)$.

Proof. The proof is the same for curves and surfaces, and we use the notations corresponding to a curve. To begin with, we perform a substitution on the error terms in the expression of $F$ :

$$
F\left(\left(A_{i}\right)_{i=0, \ldots, k}\right)=F\left(\left(B_{i}+O\left(h^{n-i+1}\right)\right)_{i=0, \ldots, k}\right)=F\left(\left(B_{i}+O\left(h^{n-k+1}\right)\right)_{i=0, \ldots, k}\right) .
$$

Since $F\left(\left(B_{i}\right)_{i=0, \ldots, k}\right)$ is a $C^{1}$ function, and denoting $D F_{p}$ the differential of $F$ at point $p$, an order one Taylor formula yields:

$$
\begin{aligned}
F\left(\left(B_{i}+O\left(h^{n-k+1}\right)\right)_{i=0, \ldots, k}\right) & \left.=F\left(\left(B_{i}\right)_{i=0, \ldots, k}\right)+D F_{\left(B_{i}+u O\left(h^{n-k+1}\right)\right)_{i=0, \ldots, k}}\left(O\left(h^{n-k+1}\right), \ldots, O\left(h^{n-k+1}\right)\right)^{t}, u \in\right] 0,1[ \\
& =F\left(\left(B_{i}\right)_{i=0, \ldots, k}\right)+O\left(h^{n-k+1}\right) .
\end{aligned}
$$




\section{Appendix to Experimental study}

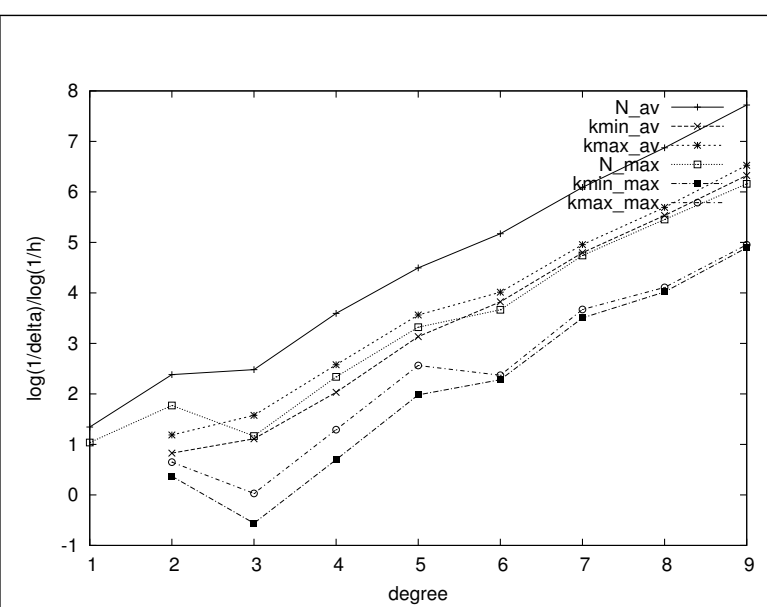

Figure 24: Exponential model: Convergence of normal and curvature estimates wrt the degree of the interpolation fitting

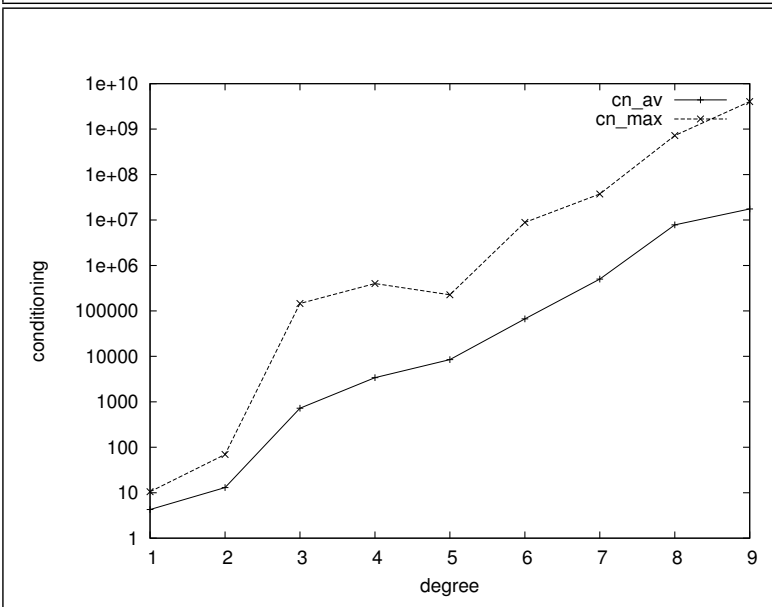

Figure 26: Exponential model: Conditioning wrt the degree of the interpolation fitting

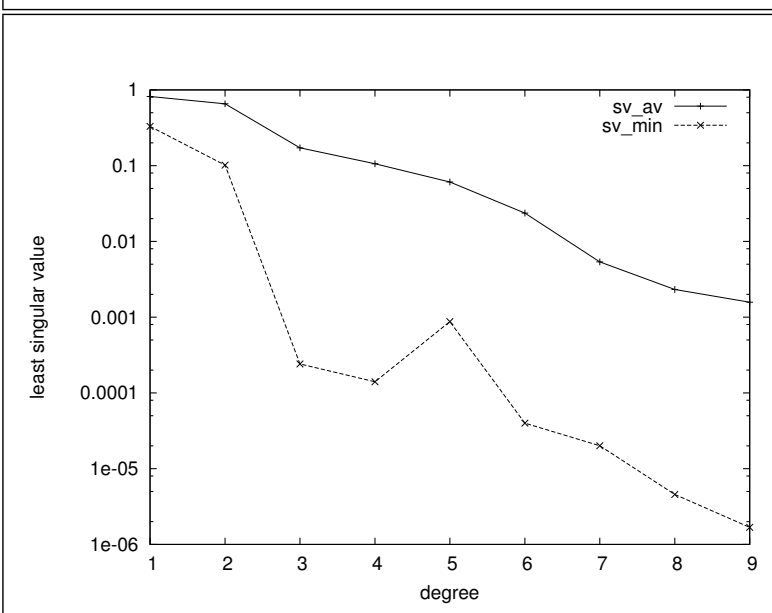

Figure 28: Exponential model: Least singular value wrt the degree of the interpolation fitting

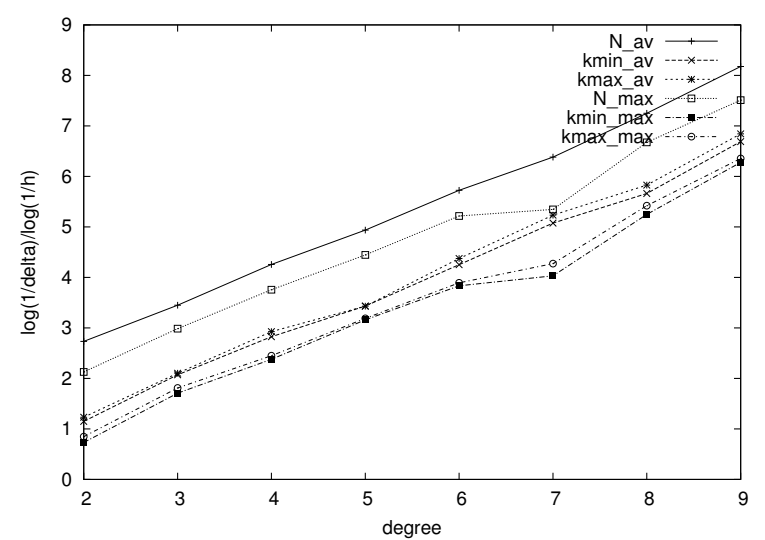

Figure 25: Exponential model: Convergence of normal and curvature estimates wrt the degree of the approximation fitting

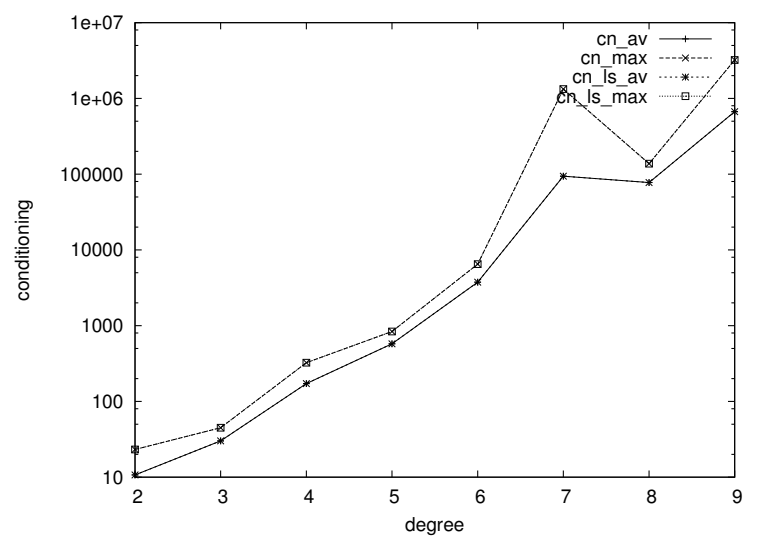

Figure 27: Exponential model: Conditioning wrt the degree of the approximation fitting

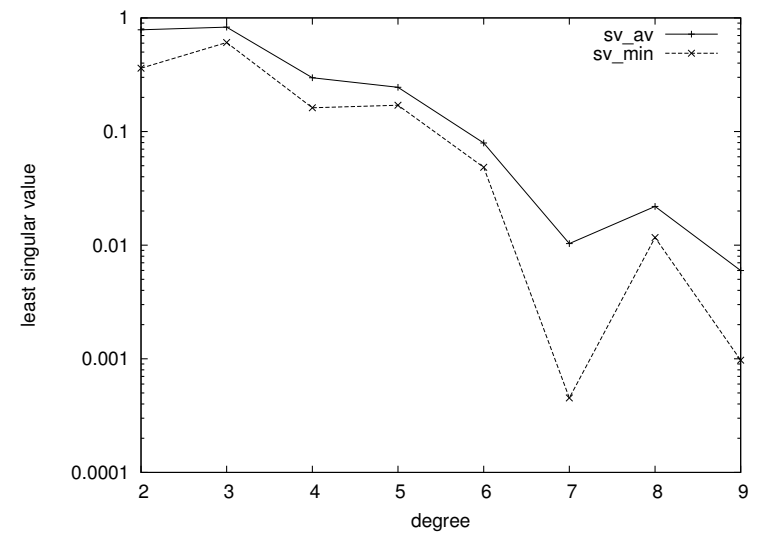

Figure 29: Exponential model: Least singular value wrt the degree of the approximation fitting 